\title{
„Gute alte Zeiten, als jedes Ding nur zwei Seiten hatte!"
}

Die Therapie der indolenten Lymphome, speziell des follikulären Lymphoms, war in den vergangenen Jahrzehnten relativ statisch. Die Erkrankung ist zwar für eine Vielzahl von Chemotherapeutika empfindlich und auch im Rezidiv noch einer Therapie zugänglich, der Langzeitverlauf blieb jedoch auch nach unterschiedlichen Therapiestrategien nahezu unbeeinflusst. Entsprechend gab und gibt es in verschiedenen nationalen und internationalen Guidelines keinen Konsensus über die beste Chemotherapie-Option beim follikulären Lymphom [Ghielmini M et al. ESMO Guidelines follicular lymphoma. Ann Oncol. 2013;24(3): 561-76)]. Erst mit der Einführung des Anti-CD20-Antikörpers Rituximab wurde endlich ein erster Fortschritt im Hinblick auf das Gesamtüberleben erreicht, die Auswahl der optimalen Chemotherapie blieb aber weiter umstritten. In der vorliegenden Ausgabe wird ausführlich ein in Deutschland bewährtes, in den USA noch häufig als innovativ bezeichnetes Therapieregime unter Einschluss von Bendamustin diskutiert.

In den 60er Jahren war Bendamustin in der DDR als günstige Alternative zu Cyclophosphamid synthetisiert worden. Die Neubewertung dieser alten Substanz ist das Verdienst von Matthias Rummel und der Studiengruppe indolente Lymphome (StiL), die Bendamustin in Kombination mit Rituximab (B-R) in direktem Vergleich mit einer Standardtherapie (R-CHOP) untersuchten. Es bestätigte sich speziell die gute Verträglichkeit von Bendamustin. Vor allem die im Alltag stark einschränkenden Nebenwirkungen Alopezie und Neuropathie waren geringer. So steht neben R-CHOP, R-CVP und anderen nun B-R als zusätzliche Behandlungsoption zur Auswahl. Auf der anderen Seite kann aufgrund aktueller Studiendaten nicht mehr zu einer Fludarabinhaltigen Primärtherapie geraten werden.

\section{Was bringt die Zukunft?}

In welche Richtung werden sich künftige Therapien entwickeln? Gerade beim follikulären Lymphom spielt die Immunabwehr eine wichtige prognostische Rolle. Patienten, in denen eine spezifische T-Zell-getriggerte Immunabwehr ausgelöst wird, haben eine deutlich bessere Prognose als Patienten mit einer (unspezifischen) Makrophagenreaktion. In Vakzinierungsstudien wurde daher versucht, diese Immunreaktion zu stimulieren, allerdings mit durchaus uneinheitlichen Ergebnissen.

Eine neue Option könnte sich durch die auch beim follikulären Lymphom durchgeführte Genomsequenzierung ergeben. Bei ca. $25 \%$ der follikulären Lymphome sind Mutationen des EZH2-Gens nachweisbar. Das EZH2-Gen greift unmittelbar in die DNS-Methylierung der malignen Zelle ein und ist damit ein möglicher therapeutischer Ansatz.

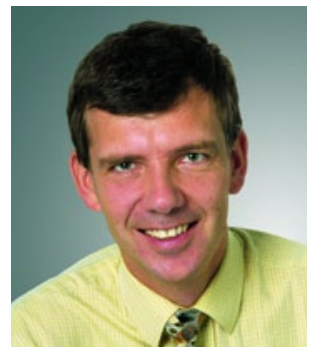

Prof. Dr. Martin Dreyling

Oberarzt an der Medizinischen Klinik und Poliklinik III des Klinikums der Universität München
Auf der anderen Seite haben gerade die immunmodulatorisch wirksamen Substanzen (IMiDs) erstmals die Notwendigkeit einer chemotherapiehaltigen Erstbehandlung beim follikulären Lymphom in Frage gestellt. In einer Phase-II-Studie wurden vergleichbare Ansprechraten und ein vergleichbares progressionsfreies Überleben erreicht wie in anderen Studien mit einer Standard-Chemotherapie (R-CHOP oder B-R) [Fowler N H et al. ASH 2012]. Derzeit wird in einer großen internationalen Studie der Wert einer chemotherapiefreien Primärtherapie beim follikulären Lymphom untersucht.

Schließlich gab es im letzten Jahr beeindruckende Ergebnisse mit Inhibitoren des B-Zellrezeptor-Signalwegs in verschiedenen Lymphom-Suptypen einschließlich der CLL und dem M. Waldenström, aber auch einem Teil der aggressiven Lymphome (Mantelzell-Lymphom und diffuses großzelliges Lymphom). Von diesem Zellsignal hängt ab, ob der Lymphozyt während seiner physiologischen Reifung im Keimzentrum des Lymphknotens überlebt, und es ist durchaus erstaunlich, dass auch die maligne Zelle noch von diesem Signalpfad abhängig ist.

All diese Therapieoptionen haben das Potential, die Standardtherapie des indolenten Lymphoms zu revolutionieren. Aggressivere Therapieoptionen wie die Stammzelltransplantation werden ins höhere Rezidiv zurücktreten. Möglicherweise ist die heutige Standard-Chemotherapie in zehn Jahren nur noch für einen Teil unserer Patienten mit indolenten Lymphom notwendig. Damit würde sich endlich ein Kreis schließen von dem langjährigen Wissen um die molekulargenetische Entstehung des indolenten Lymphoms und der zentralen Rolle der immunologischen Tumorabwehr hin zu einer gezielteren, und damit wirksameren, aber auch besser verträglicheren Therapie der indolenten Lymphome.

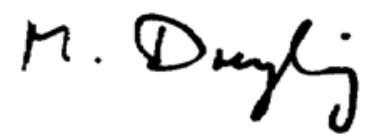

